\title{
Single Channel Analysis of Isoflurane and Ethanol Enhancement of Taurine-Activated Glycine Receptors
}

\author{
Dean Kirson, ${ }^{1}$ Jelena Todorovic, ${ }^{1}$ and S. John Mihic \\ Department of Neuroscience, Division of Pharmacology and Toxicology, Waggoner Center for Alcohol \& Addiction Research, \\ Institutes for Neuroscience and Cell \& Molecular Biology, University of Texas at Austin, Austin, Texas
}

Received July 8, 2017; accepted October 26, 2017

\section{ABSTRACT}

The amino acid taurine is an endogenous ligand acting on glycine receptors (GlyRs), which is released by astrocytes in many brain regions, such as the nucleus accumbens and prefrontal cortex. Taurine is a partial agonist with an efficacy significantly lower than that of glycine. Allosteric modulators such as ethanol and isoflurane produce leftward shifts of glycine concentration-response curves but have no effects at saturating glycine concentrations. In contrast, in whole-cell electrophysiology studies these modulators increase the effects of saturating taurine concentrations. A number of possible mechanisms may explain these enhancing effects, including modulator effects on conductance, channel open times, or channel closed times. We used outside-out patch-clamp single channel electrophysiology to investigate the mechanism of action of $200 \mathrm{mM}$ ethanol and $0.55 \mathrm{mM}$ isoflurane in enhancing the effects of a saturating concentration of taurine. Neither modulator enhanced taurinemediated conductance. Isoflurane increased the probability of channel opening. Isoflurane also increased the lifetimes of the two shortest open dwell times while both agents decreased the likelihood of occurrence of the longest-lived intracluster channel-closing events. The mechanism of enhancement of GlyR functioning by these modulators is dependent on the efficacy of the agonist activating the receptor and the concentration of agonist tested.

\section{Introduction}

Although glycine receptors (GlyRs) are responsible for most inhibitory neurotransmission in the brainstem and spinal cord, these receptors are also found in higher brain regions such as the hippocampus, nucleus accumbens, and prefrontal cortex (van den Pol and Gorcs, 1988; Malosio et al., 1991; Lynch, 2004; Waldvogel et al., 2007; Baer et al., 2009; Jonsson et al., 2009, 2012; Lu and Ye, 2011). GlyRs are members of the Cys-loop ligand-gated ion channel superfamily, and each receptor is comprised of five subunits that coassemble around a central anion-conducting pore. Many different compounds modulate GlyR function, including zinc, alcohols, and volatile anesthetics (Lynch, 2004; Yevenes and Zeilhofer, 2011). Some of the behavioral effects of these compounds are thought to be mediated by GlyR, as would be expected given GlyR subunit expression in the brain regions important for these effects (Yamashita et al., 2001; Molander et al., 2005, 2007; Badanich et al., 2013).

Alcohols and volatile anesthetics enhance GlyR-mediated currents in a concentration-dependent manner (Mascia et al., 1996a,b), producing leftward shifts of glycine concentrationresponse curves, with no effects on currents generated by maximally effective concentrations of glycine (Welsh et al., 2010; Kirson et al., 2012). At the single channel level, ethanol

This research was supported by a Bruce Jones predoctoral fellowship and the Waggoner Center for Alcohol \& Addiction Research.

${ }^{1}$ D.K. and J.T. contributed equally to this research.

https://doi.org/10.1124/jpet.117.243840. enhancement of currents produced by submaximal glycine concentrations is due to ethanol decreasing glycine unbinding rates, with no changes in open probability $\left(P_{\text {open }}\right)$; i.e., ethanol increases burst durations (Eggers and Berger, 2004; Welsh et al., 2009).

The sulfonic acid taurine acts as a partial agonist of the GlyR, producing $~ 50 \%$ of the current produced by maximally effective concentrations of glycine (Lape et al., 2008). Taurine is the second most abundant amino acid in the brain, and taurine release leads to GlyR activation (Dahchour et al., 1996; Mori et al., 2002; Albrecht and Schousboe, 2005; Ericson et al., 2006; Choe et al., 2012). GlyRs have been implicated in mediating the effects of multiple drugs of abuse, and interestingly taurine-mediated GlyR activation plays a role in the rewarding effects of ethanol (Molander et al., 2005, 2007; Ericson et al., 2011; Jonsson et al., 2014). Unlike glycineactivated GlyR currents, taurine-mediated currents measured on the whole-cell level are enhanced by many allosteric modulators when tested with maximally effective concentrations of taurine (Kirson et al., 2012, 2013). We hypothesized that these modulators would increase $P_{\text {open }}$ on taurineactivated GlyR, which could be due to increased mean channel open times and/or decreased mean channel closed times.

Here, we report on studies conducted on human $\alpha 1$ homomeric GlyR expressed in Xenopus oocytes, investigating various single channel parameters for changes in taurinemediated GlyR activation by ethanol and the volatile anesthetic isoflurane. We examined channel conductance, open and closed dwell times, and cluster lengths, as well as 
determining the probabilities of channel opening in the absence and presence of these two modulators.

\section{Materials and Methods}

Reagents. Xenopus laevis were purchased from Nasco (Ft. Atkinson, WI) and housed at $19^{\circ} \mathrm{C}$ on a 12-hour light:dark cycle. Animals were housed, and surgeries performed, in accordance with Association for Assessment and Accreditation of Laboratory Animal Care regulations (https://www.aaalac.org/). All reagents were purchased from SigmaAldrich (St. Louis, MO) except isoflurane, which was obtained from Anaquest (New Providence, NJ). BioUltra taurine (Sigma-Aldrich) was used in all experiments.

Single Channel Electrophysiology Recording. Oocyte isolation, GlyR subunit cDNA nuclear injection, and oocyte storage are described in Kirson et al. (2012). Outside-out patches were pulled from the animal poles of Xenopus oocytes expressing wild-type $\alpha 1$ homomeric GlyRs. Before recording, each oocyte was placed in a highosmolarity stripping solution ( $200 \mathrm{mM}$ sodium methyl sulfate, $20 \mathrm{mM}$ $\mathrm{KCl}, 10 \mathrm{mM} \mathrm{HEPES}$, and $1 \mathrm{mM} \mathrm{MgCl}_{2}$ ) to aid in manual removal of the vitelline membrane using forceps. Patch pipettes were pulled from thick-walled borosilicate glass (WPI, Sarasota, FL) using a P-97 Flaming/Brown Micropipette Puller (Sutter Instruments, Novato, CA). Pipettes were fire polished with an MF-830 Microforge (Narishige, Tokyo, Japan) to obtain a smooth tip, and those with resistances of 5-15 $\mathrm{M} \Omega$ were used in recordings. Outside-out patches were held at $-80 \mathrm{mV}$, and recordings were made according to standard methods (Hamill et al., 1981) using an Axopatch 200B amplifier (Molecular Devices, Sunnyvale, CA). Data were digitized at $100 \mathrm{kHz}$ with a Digidata 1322A (Molecular Devices), low-pass filtered at $10 \mathrm{kHz}$, and recorded on a computer hard drive using pClamp 9 software (Molecular Devices). Taurine and drug solutions were prepared in external solution $(100 \mathrm{mM} \mathrm{NaCl}, 2 \mathrm{mM} \mathrm{KCl}, 1 \mathrm{mM}$ $\mathrm{MgCl}_{2} \cdot 6 \mathrm{H}_{2} \mathrm{O}, 10 \mathrm{mM}$ HEPES, $2 \mathrm{mM} \mathrm{CaCl}_{2}$ at $\left.\mathrm{pH} 7.4\right)+2.5 \mathrm{mM}$ tricine ( $N$-[2-hydroxy-1,1-bis(hydroxymethyl)ethyl]glycine), to chelate free zinc before being perfused over outside-out patches using an SF-77B Perfusion Fast Step apparatus (Warner Instruments, Hamden, CT). The pipette internal solution contained $102 \mathrm{mM} \mathrm{CsCl}, 10 \mathrm{mM}$ HEPES, $2 \mathrm{mM} \mathrm{MgCl}{ }_{2} \cdot 6 \mathrm{H}_{2} \mathrm{O}, 10 \mathrm{mM}$ EGTA, and $1 \mathrm{mM} \mathrm{CaCl}_{2}$ at $\mathrm{pH} 7.2$.

Data Analysis. Data were analyzed using the single channel analysis programs in $\mathrm{QuB}$ (Qin et al., 2000a,b); version 2.0.0.9 was used for preprocessing, open/closed dwell-time analysis and chopping of data into clusters. Tracings were first baseline corrected to correct for drift and portions of the tracings that showed multiple channels activating simultaneously were excised. Portions of tracings clearly representing closed or open channel states were selected by eye and fit to Gaussian curves using the amplitude modeling subroutine in $\mathrm{QuB}$, to determine currents representing closed and open states in each patch. The conductance in each patch was determined by subtracting the mean closed current from the mean open current and then dividing that number by the holding voltage $(-80 \mathrm{mV})$. Channel opening and closing events were idealized using the segmental- $k$-means algorithm (Qin et al., 2000a,b). Data were initially idealized with a simple twostate closed-to-open $(\mathrm{C} \leftrightarrow \mathrm{O})$ model. Multiple closed and open states were then added sequentially to form a star model (closed state as the center) using the maximum interval likelihood fitting method, after imposing a dead time resolution of 30 microseconds. When this model was complete, as determined by lack of statistically significant increases in log likelihood seen after adding additional closed and open states, the data were re-idealized and used for open and closed channel dwell-time analyses. Durations of opening and closing events were sorted into 50 bins each, based on individual channel opening or closing durations. This allowed for the creation of dwell-time distributions, constructed using the log time on the abscissa and the square root of the number of events per bin divided by total events on the ordinate. These data were then fit with a mixture of exponential components using the maximum interval likelihood function in $\mathrm{QuB}$.
Clusters were defined as being separated by closed-time durations equal to or longer than the longest $\tau_{\text {crit }}$ value determined in each patch. Four closed time distributions were fit to the data. The longest and rarest ( $\sim 0.12 \%$ prevalence) closed time was the most variable among patches in terms of lifetime when taurine was applied alone, with ethanol, or with isoflurane, and was consequently considered as occurring between clusters. The $\tau_{\text {crit }}$ values determined between the third- and fourth-longest closed states in each patch were used to chop data into individual clusters; these $\tau_{\text {crit }}$ values ranged from $6.2 \pm 1.2$ to $9.2 \pm 1.1$ milliseconds among the three treatment groups. Once idealized, data were chopped into clusters, and the clusters were saved in a separate file for analysis using a kinetic model involving agonist-bound closed, flipped, and open states (Lape et al., 2008).

Statistical analyses were performed using SigmaPlot version 11.0 (Systat Software, San Jose, CA) utilizing one-way analysis of variance and Bonferroni $t$ test post hoc comparisons.

\section{Results}

Ethanol and Isoflurane Effects on Taurine-Activated GlyR Single Channel Conductance. Single channel recording experiments were conducted on outside-out patches pulled from Xenopus oocytes expressing the human homomeric $\alpha 1$ GlyR. Patches were exposed to $200 \mathrm{mM}$ ethanol or $0.55 \mathrm{mM}$ isoflurane coapplied with $100 \mathrm{mM}$ taurine, and the data obtained were compared with patches exposed to $100 \mathrm{mM}$ taurine alone. This saturating concentration of taurine was chosen to maximize channel opening (Lape et al., 2008). Taurine applied alone, or in combination with one of the modulators, produced clearly defined clusters of channel activity, as shown in Fig. 1. There was a significant effect of experimental condition on conductance $[F(2,18)=5.24$, $P<0.02$ ], with the conductance seen in the presence of ethanol being slightly lower than that of the taurine-alone control (Fig. 2). Isoflurane had no effect on conductance.

Isoflurane Increases Taurine-Activated GlyR Mean Open Time. When a saturating concentration of taurine was applied alone, GlyR exhibited a mean open time of $0.63 \pm 0.04$ milliseconds $(n=8)$. The mean open time was determined by summing the products of the three open dwell times and likelihoods in each patch and was significantly increased by isoflurane but not ethanol $[F(2,18)=8.45, P<0.003]$ (Fig. 3A). The closed mean time was calculated by summing the products of the three shortest closed dwell times and likelihoods in each patch. Both ethanol and isoflurane appeared to decrease the mean closed time, but not significantly (Fig. 3B).

Isoflurane Increases the Lifetimes of the First Two Open Times. Open dwell-time $(\tau)$ data were adequately described using three exponential components for all three experimental conditions, since the addition of extra open states did not significantly improve fits of data. In two out of eight taurine control patches, as well as three out of five taurine + isoflurane patches, the longest open-time component $(\tau 3)$ could not be fit. Sample open dwell-time histograms from individual patches are shown in Fig. 4A for all three experimental conditions. The $\tau$ values correspond to the times at which peaks were observed for the three exponential functions fit in Fig. 4A. One-way analysis of variance revealed that isoflurane, but not ethanol, significantly increased the lifetimes of the briefest $[F(2,18)=5.6, P<0.015]$ as well as the next shortest $[F(2,18)=9.66, P<0.002]$ open times $(\tau 1$ and $\tau 2$ in Fig. 4B). Ethanol and isoflurane were also tested for their effects on the likelihoods of opening (amplitude) to these dwell 


\section{A \\ $100 \mathrm{mM}$ Taurine
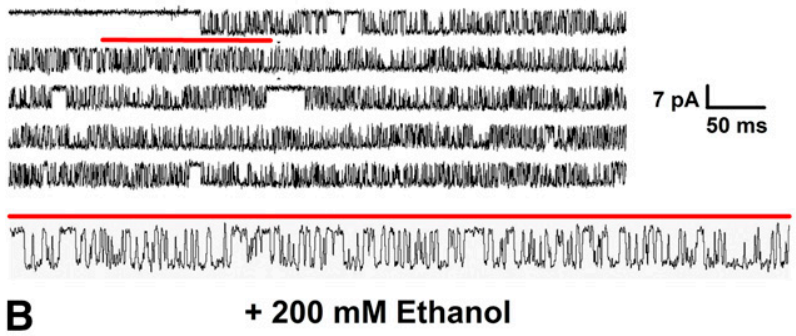

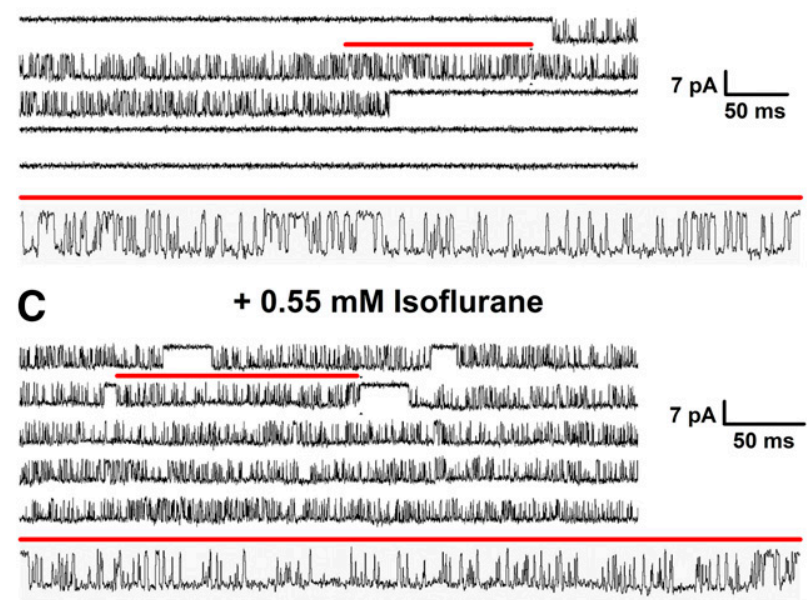

Fig. 1. Sample tracings of taurine-activated GlyR alone and with coapplied ethanol or isoflurane. Representative tracings show $\sim 2-3$ seconds of single channel homomeric $\alpha 1$ GlyR responses to $100 \mathrm{mM}$ taurine (A), $100 \mathrm{mM}$ taurine $+200 \mathrm{mM}$ ethanol (B), or $100 \mathrm{mM}$ taurine + $0.55 \mathrm{mM}$ isoflurane (C). The horizontal red line above a portion of each top trace denotes the $\sim 150$ millisecond expanded view seen in the trace below.

times (Fig. 4C); this refers to the areas under the curves of the three dwell times, as shown in the sample histograms in Fig. 4A. No effects of ethanol or isoflurane were seen on amplitude.

Ethanol and Isoflurane Have No Effects on Channel Intracluster Closed Dwell-Time Components but Do Affect Closed Likelihoods. Intracluster closed channel dwell times were fit by three exponential components $(\tau \mathrm{s})$ for all three experimental conditions tested. A fourth (much longer and rare) closed time that varied considerably in time between patches was considered to represent closings seen between clusters. Sample closed dwell-time histograms for all conditions can be seen in Fig. 5A. There were no significant effects of ethanol or isoflurane on the durations of $\tau 1, \tau 2$, or $\tau 3$ (Fig. 5B). Neither ethanol nor isoflurane affected the likelihoods of occurrence of the two shorter closed dwell-time components (Fig. 5C); however, both agents decreased the likelihood of observing the longest-lived intracluster dwell time $[F(2,18)=8.23, P<0.003]$ (Fig. $5 \mathrm{C}$ ).

Mechanistic Fits to Intra-Cluster Data. Lape et al. (2008) proposed that the partial agonism at glycine and nicotinic acetylcholine receptors was not due to a decreased efficacy $(E)$ of partial agonists compared with higher efficacy agonists, as had been previously thought. The $E$ value is defined as the rates of transition from closed to open states $(\beta)$ divided by the transition rates from open to closed states $(\alpha)$. Lape et al. (2008) showed that the $E$ values of glycine and taurine were quite similar. Instead, agonists varied in their abilities to transition the receptor from an agonist-bound closed state to another preopen closed state, which they termed flipped (Lape et al., 2008).

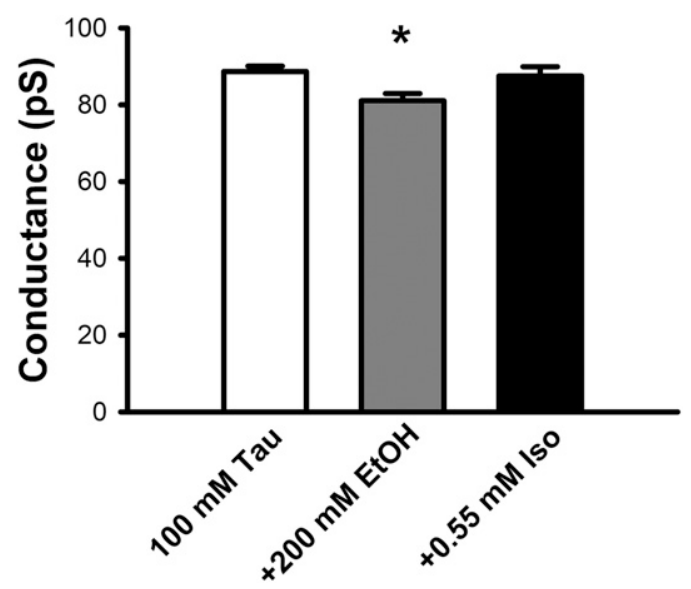

Fig. 2. Ethanol and isoflurane effects on taurine-activated GlyR conductance. The conductance $(\mathrm{pS})$ is graphed for each experimental condition. Taurine-activated GlyR conductance was $88.7 \pm 1.4 \mathrm{pS}(n=8$ patches; 368,347 total events) as seen in the white bar. Ethanol (gray bar) slightly decreased conductance to $81.1 \pm 1.9 \mathrm{pS}$ ( $n=6$ patches; $1,075,247$ total events) while conductance in the presence of isoflurane was $87.5 \pm 2.4 \mathrm{pS}$, respectively ( $n=5$ patches; 222,663 total events). Data are shown as mean + S.E.M. of $5-8$ patches. ${ }^{*} P<0.05$.

The transition rate from closed to flipped states $(\delta)$ divided by the converse $(\gamma)$ was defined as $F$, and this differed markedly between glycine and taurine. We reasoned that, at a concentration of $100 \mathrm{mM}$ taurine, receptors would be found in the triply liganded state within clusters and that the intracluster behavior could be modeled as transitions among closed $\left(A_{3} R\right)$, flipped $\left(A_{3} F\right)$, and open $\left(A_{3} R^{*}\right)$ states. Figure $6 \mathrm{~A}$ shows the transition rates calculated in the absence and presence of allosteric modulators using the kinetic model depicted. Isoflurane, but not ethanol, increased the $\delta[F(2,18)=4.62, P<0.03]$ and $\beta[F(2,18)=9.1, P<0.002]$ transition rates, decreased $\alpha[F(2,18)$ $=8.42, P<0.003]$, and had no effect on $\gamma$ (Fig. 6A) compared with taurine alone. This led to isoflurane-induced increases in both $F[F(2,18)=5.62, P<0.014]$ (Fig. 6B) and $E[F(2,18)=$ 10.42, $P<0.001$ ] (Fig. 6C). In a mechanistic model of channel activation involving a second closed state (flipped), channel opening involves transitions between closed and flipped, as well as flipped and open states, as shown in Fig. 6A. Thus the traditional method of defining efficacy as $\beta / \alpha$ is inadequate at GlyR since the $\delta$ and $\gamma$ transition rates between closed and flipped states, yielding $F=\delta / \gamma$, must also be taken into account. Lape et al. (2008) defined effective efficacy $\left(E_{\text {eff }}\right)$ to combine $F$ and $E$ such that $E_{\text {eff }}=E F /(1+F)$. This $E_{\text {eff }}$ value was more than doubled by isoflurane but not affected significantly by ethanol $[F(2,18)=14.81, P<0.001]$ (Fig. 6D). The likelihoods of channels being found in the closed, flipped, or open states were also calculated from the transition rates. Isoflurane increased $P_{\text {open }}[F(2,18)=8.89, P<0.003]$ (Fig. 7A) and decreased $P_{\text {closed }}[F(2,18)=6.56, P<0.008]$ (Fig. 7B), but had no effect on $P_{\text {flipped }}$ (Fig. 7C). The average open (Fig. 7D), closed (Fig. 7E), and flipped (Fig. 7F) state lifetimes were also determined. Ethanol had no effect on any state lifetime but isoflurane did increase the open state lifetime $[F(2,18)=$ $10.25, P<0.001]$.

\section{Discussion}

In the present report we examined the effects of anesthetically relevant concentrations of ethanol and isoflurane for 
A

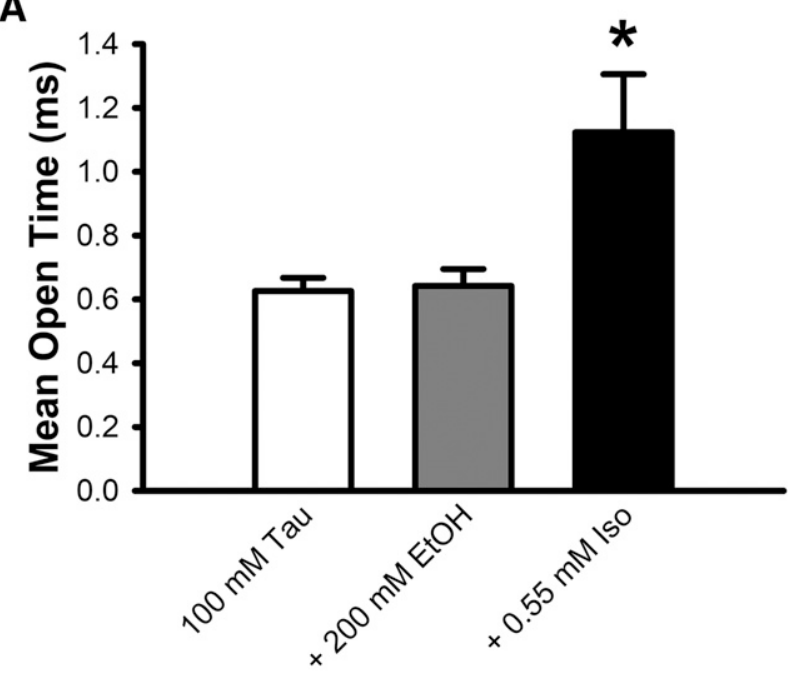

B

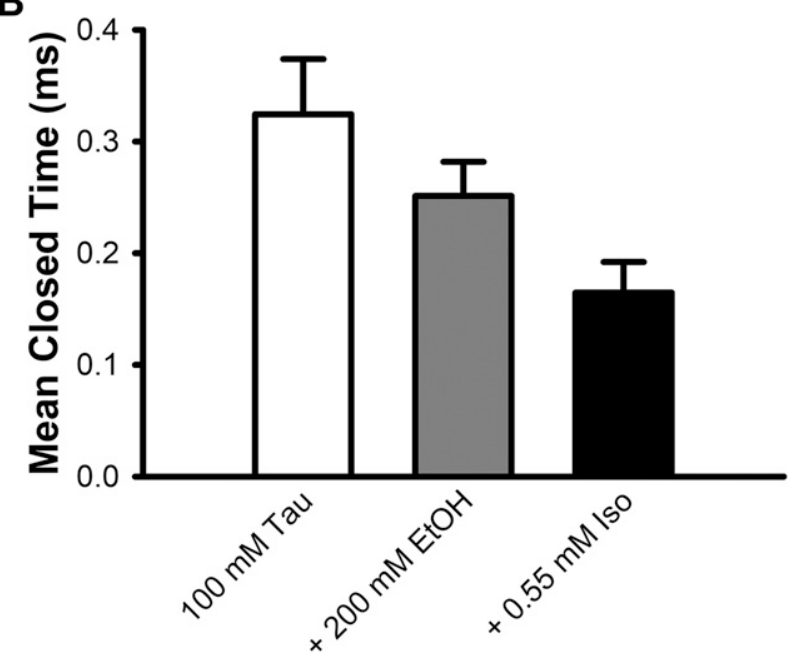

Fig. 3. Isoflurane significantly increases mean open time. (A) Summary graph showing the effects of ethanol (gray bar) and isoflurane (black bar) on mean open times of the taurine-activated GlyR. Only isoflurane significantly increased mean open time of channels. (B) Summary graph showing the effects of ethanol (gray bar) and isoflurane (black bar) on mean closed times of the taurine-activated GlyR. Neither isoflurane nor ethanol significantly decreased mean closed time of channels. Data are shown as mean + S.E.M. of $5-8$ patches. ${ }^{*} P<0.05$.

their effects on taurine-activated GlyR. The concentration of isoflurane used $(0.55 \mathrm{mM})$ is slightly less than twice the $\mathrm{EC}_{50}$ value of isoflurane in mammals $(0.32 \mathrm{mM})$ and would be expected to anesthetize essentially all subjects (Franks and Lieb, 1994). A concentration of $200 \mathrm{mM}$ ethanol is equivalent to a blood alcohol level of $920 \mathrm{mg} \%$, which would be expected to be either anesthetizing or lethal depending on a subject's level of functional tolerance. We chose these concentrations because larger modulator effects would make it easier to detect more subtle drug responses.

We previously showed that these allosteric modulators studied on the whole-cell level enhance the effects of saturating concentrations of taurine, but not glycine, on $\alpha 1$ GlyR function (Kirson et al., 2012, 2013). However, in these studies we were not able to distinguish among the possible mechanisms of action of these compounds. One, admittedly unlikely, possibility is that modulators could enhance taurine-mediated but not glycine-mediated GlyR conductance, which would explain why they enhance the effects of saturating concentrations of the former but not the latter. The data shown in Fig. 2 disprove that hypothesis; if anything, ethanol slightly decreased conductance. Another possibility is that modulators could affect taurine affinity, but this would not be expected to be the case when saturating concentrations of taurine are used. Under saturating agonist conditions, the unbinding of an agonist molecule would almost immediately be followed by another agonist binding event, preventing modulators from exerting their effects via changes in agonist affinity. A third possible explanation is that modulators increase the channel $P_{\text {open, }}$ after activation by taurine but not glycine, since the latter produces a $P_{\text {open }}$ value near 0.95 at saturating concentrations. This increase in taurine's $P_{\text {open }}$ value could be accomplished in four different ways, as follows: 1) by increasing channel open lifetimes $(\tau \mathrm{s}) ; 2$ ) by decreasing channel closed lifetimes; 3 ) by increasing the likelihoods (amplitude) of longer-lived open states; or 4) by decreasing the likelihoods of longer-lived closed states. In the present report we show that significant increases in the two shorter time constants of opening events were found after application of isoflurane (Fig. 4B) but not ethanol. In addition, both ethanol and isoflurane decreased the likelihood of occurrence of the longest-lived closed state (see amp3 in Fig. 5C). Both of these findings would be consistent with an increase in the $P_{\text {open }}$ value in the presence of a modulator; therefore, it is likely that both of these phenomena contribute to the overall increase in the

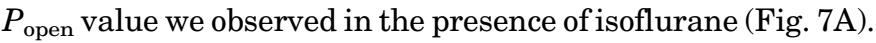

After chopping our data into clusters we fit the intracluster opening and closing events to a mechanistic model equivalent to the fully liganded flipped model described by Lape et al. (2008), in which three taurine molecules are bound; we believe this to be a reasonable model since the receptor was exposed to a saturating concentration of $100 \mathrm{mM}$ taurine. The rate constants we determined for $\alpha, \beta, \gamma$, and $\delta$ (Fig. 6A) were generally quite similar to those found for taurine by Lape et al. (2008). Their values for the GlyR that had bound three taurine molecules were $14,500(\alpha), 133,000(\beta), 5170(\gamma)$, and $740(\delta)$; only in the $\delta$ values did our numbers differ markedly. In addition, we obtained similar numbers when calculating the $F(0.29$ vs. $0.15)$ and $E$ (8.8 vs. 9.2) values. The calculated values for $P_{\text {open, }}$, $P_{\text {closed }}, P_{\text {flipped }}$, and their respective lifetimes, were also generally close to those determined by Lape et al. (2008). Ethanol and isoflurane were then tested for their effects on these different single channel properties. Isoflurane had several effects that would all tend to increase GlyR function: an increased open channel lifetime (Fig. 7D), which is a reflection of the decreased $\alpha$ value (Fig. 6A), and a decreased likelihood that the channel would be found in the closed state (Fig. 7B), since isoflurane increased the $\delta$ value (Fig. 6A). These effects would be expected to contribute to the increase seen in channel $P_{\text {open }}$ (Fig. 7A). Isoflurane enhanced transitions from the closed to the flipped states as well as to the open states (Fig. 7, B and C) and both of these contributed to the increase in the effective efficacy of taurine (Fig. 7D). Although ethanol at times trended toward having an effect, particularly on closed times, it generally was without effect. In previous whole-cell studies (Kirson et al., 2012, 2013) we found that isoflurane and other volatile anesthetics produce greater enhancement of the effects of maximally effective concentrations of taurine than does $200 \mathrm{mM}$ ethanol, and this likely explains why we saw more of an effect with 
A

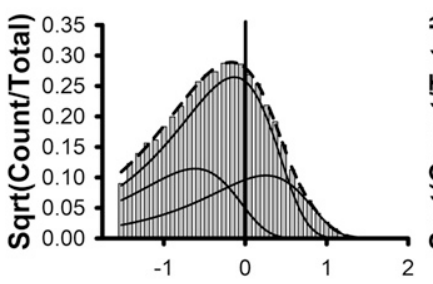

Log Duration (ms)
$+200 \mathrm{mM} \mathrm{EtOH}$
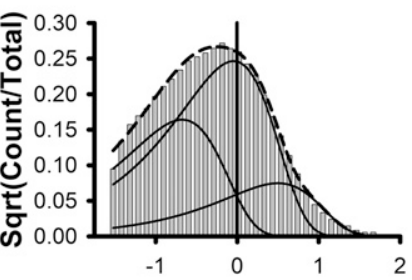

Log Duration (ms)

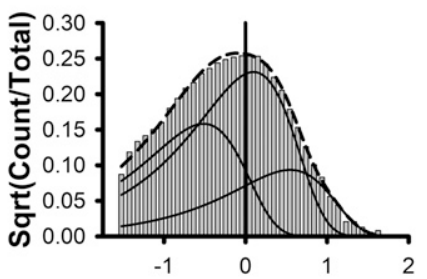

Log Duration (ms)
B

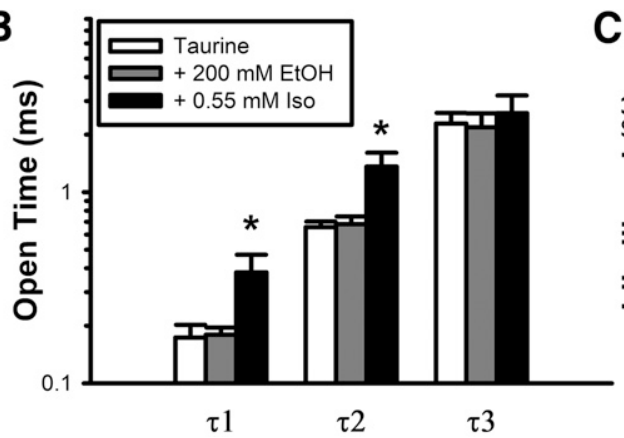

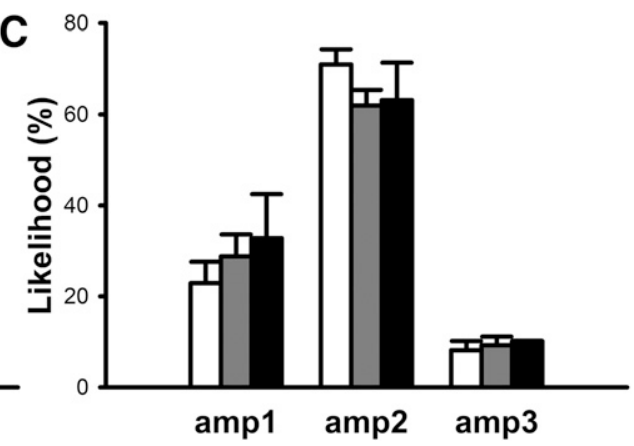

Fig. 4. Ethanol and isoflurane effects on lifetimes and likelihoods of channel opening events. (A) Representative histograms demonstrating the fits for the open dwell times. Each condition was fit using three open-time components $(\tau \mathrm{s})$. In each graph, the solid lines describe the individual dwell-time exponential functions and the dashed line is a fit of all the data. The vertical lines denote the 1 millisecond time point of open times to better illustrate differences of fit among experimental groups. (B) Only isoflurane significantly increased the average durations of the two shortest open dwell times. (C) Neither ethanol nor isoflurane affected the likelihoods of observing specific open times. Data are shown as mean + S.E.M. of 2-6 patches; the longest open times could be fit in six of the eight taurine-alone and two of the five taurine + isoflurane patches. $* P<0.05$ isoflurane on the single channel level. In several instances ethanol appeared to be having effects similar to those of isoflurane (e.g., Figs. 3B and 6B), but not great enough to be statistically different from the taurine-alone control.

In our previous single channel investigations into the effects of ethanol on the glycine-activated GlyR, only subsaturating glycine concentrations were examined and no significant changes in the $P_{\text {open }}$ value or open times were seen, although there was a trend toward an increase in mean open time in the presence of $200 \mathrm{mM}$ ethanol. Under those conditions ethanol instead exerted its potentiating effects by decreasing the rate of glycine unbinding (Eggers and Berger, 2004; Welsh et al.,
A

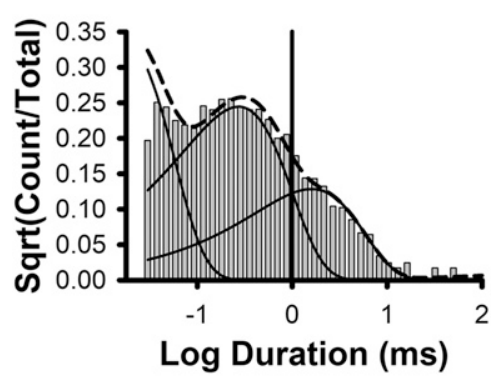

$+200 \mathrm{mM} \mathrm{EtOH}$

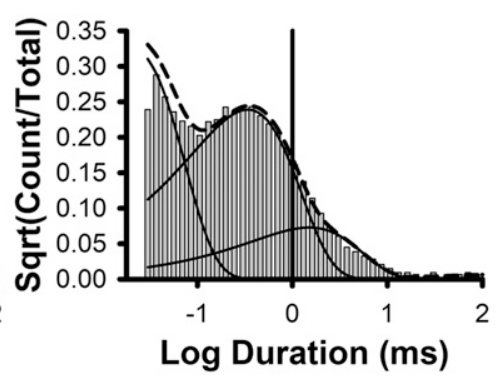

$+0.55 \mathrm{mM}$ Iso

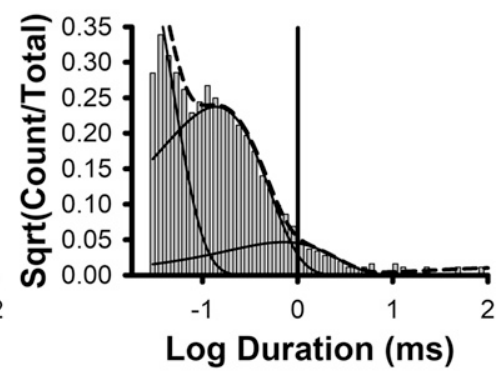

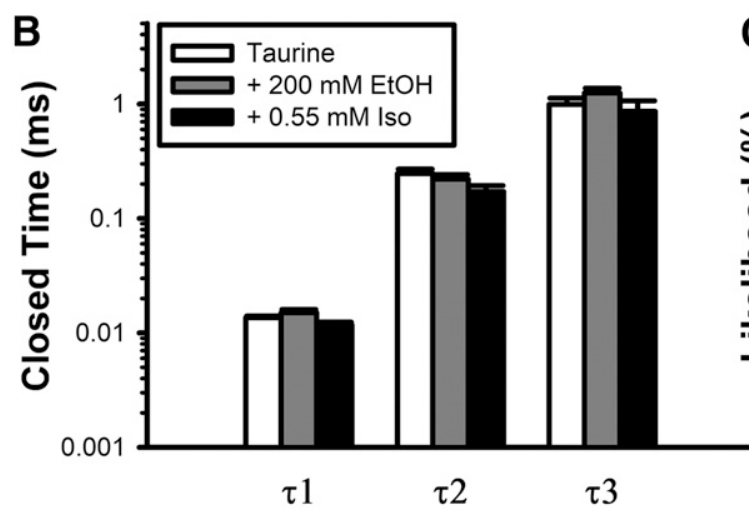
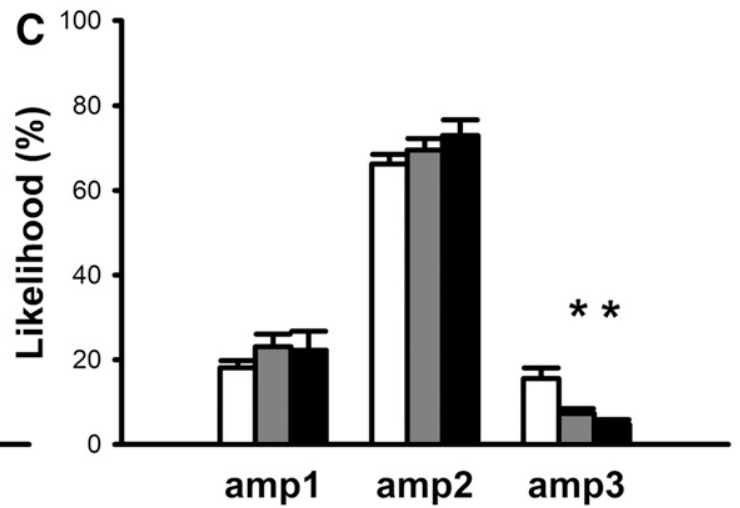

Fig. 5. Ethanol and isoflurane effects on lifetimes and likelihoods of channel-closing events. (A) Representative histograms demonstrating the fits for the closed dwell times. Each condition was fit using three closed-time components $(\tau \mathrm{s})$. In each graph, the solid lines describe the individual dwell-time exponential functions and the dashed line is a fit of all the data. The vertical lines denote the 1 millisecond time point of open times to better allow for comparisons among experimental groups. (B) Neither modulator affected average durations of closed dwell times. (C) Ethanol and isoflurane both decreased the likelihood of occurrence of the longest-lived closed-time component. Data are shown as mean + S.E.M. of 5-8 patches. 
A

$\delta$

100 mM taurine $\quad A_{3} R \underset{\substack{13,168 \pm 803 \\ \gamma}}{\stackrel{3,678 \pm 380}{\underset{1}{\rightleftarrows}}} A_{3} F \underset{\substack{11,300 \pm 298 \\ \alpha}}{\stackrel{98,033 \pm 2,673}{\rightleftarrows}} A_{3} R^{*}$

taurine $+\mathrm{EtOH}$

$A_{3} R \underset{10,773 \pm 983}{\stackrel{3,786 \pm 438}{\underset{10}{\rightleftarrows}}} A_{3} F \underset{11,795_{ \pm} 457}{\stackrel{94,292 \pm 3,353}{\rightleftarrows}} A_{3} R^{*}$

taurine + iso

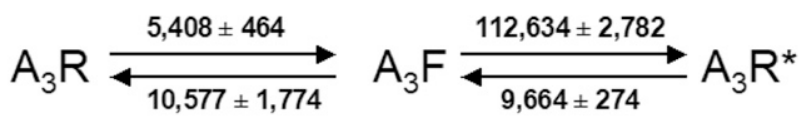

B

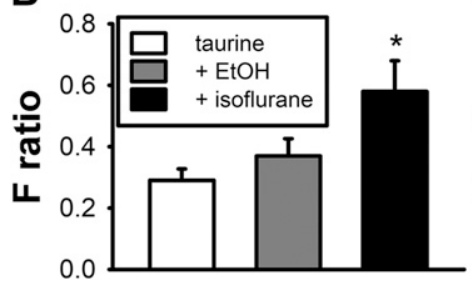

C

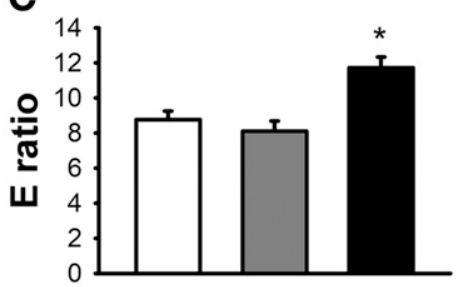

D

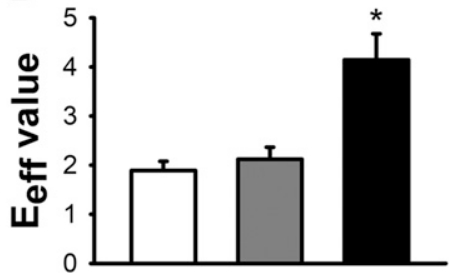

Fig. 6. Application of the flipped kinetic model to ethanol and isoflurane effects on taurine-activated GlyR. (A) Clusters of single channel recordings were isolated and fit to a kinetic scheme involving two closed states $\left(A_{3} R\right.$ and $\left.A_{3} F\right)$ as well as a single open state $\left(A_{3} R^{*}\right)$. The numbers over and under the arrows represent the mean \pm S.E.M. of the rate constants describing transitions among states. The top scheme represents $100 \mathrm{mM}$ taurine alone and the Greek symbols identify the relevant transition rates. (B) The $F$ ratio is a unitless number describing transitions between closed and flipped states $(F=\delta / \gamma)$ and was significantly increased by isoflurane. The $F$ values were determined for each patch and averaged. (C) The $E$ ratio is also unitless and describes transitions between flipped and open states $(E=\beta / \alpha)$, and was significantly increased by isoflurane. The $E$ values were determined for each patch and averaged. (D) The effective efficacy $\left(E_{\text {eff }}\right)$ is a function of $E$ and $F$ as described in Results and was significantly enhanced by isoflurane but not ethanol. Data are shown as mean + S.E.M. of $5-8$ patches. ${ }^{*} P<0.05$.

2009). Thus, at the single channel level, ethanol and isoflurane enhancement of GlyR functioning is dependent on which agonist activates the receptor and at what concentration that agonist is tested.
In summary, we investigated at the single channel level the effects of ethanol and isoflurane on saturating concentrations of taurine applied to the GlyR in isolated outside-out patches. Isoflurane increased the average lifetimes of the two shortest
A

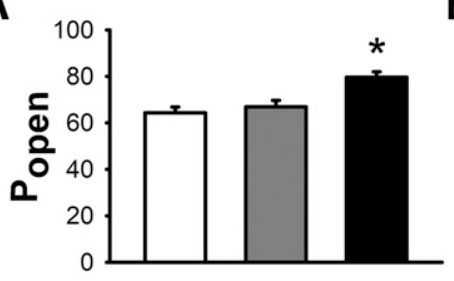

D

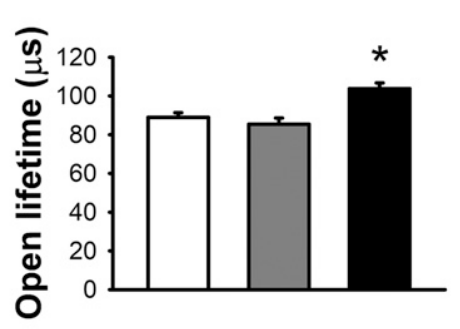

B

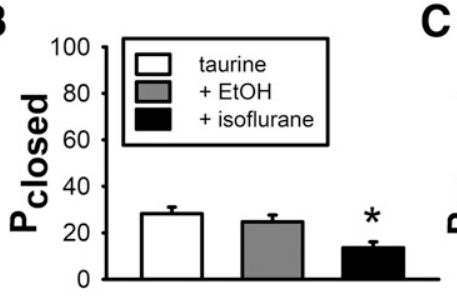

E

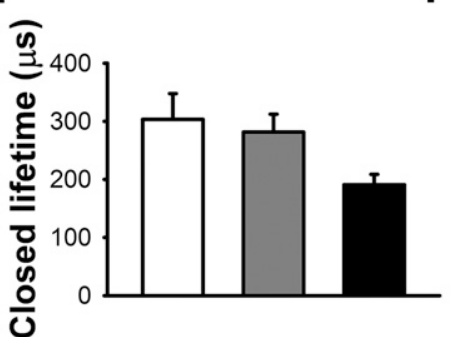

C

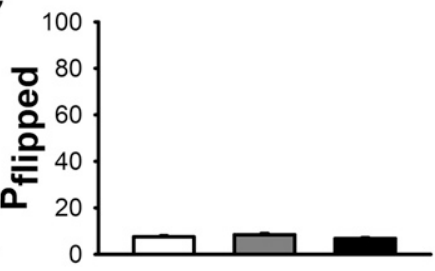

$\mathbf{F}$

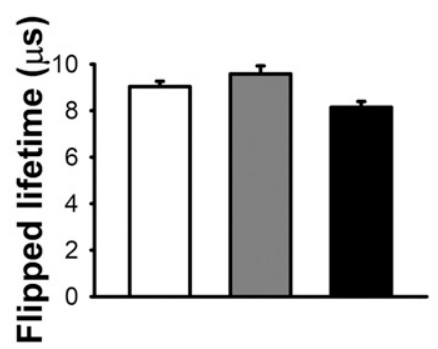

Fig. 7. Ethanol and isoflurane effects on open and closed likelihoods and lifetimes determined from the flipped kinetic model. (A-C) The probabilities of channels being found in open (A), closed (B), and flipped (C) states were determined in the absence and presence of allosteric modulators. In each patch $P_{\text {open }}$ was determined as $(\beta / \alpha \times \delta / \gamma) /(1+\beta / \alpha+\beta / \alpha \times \delta / \gamma) \times 100$, while $P_{\text {closed }}$ was defined as $(\alpha / \beta \times \gamma / \delta) /(1+\alpha / \beta+\alpha / \beta \times \gamma / \delta) \times 100$ and $P_{\text {flipped }}=100-$ $P_{\text {open }}-P_{\text {closed. }}$. Isoflurane enhanced $P_{\text {open }}$ and decreased $P_{\text {closed. }}$. No effects on $P_{\text {flipped }}$ were noted. (D-F) The lifetimes of open, closed, and flipped states were determined in the absence and presence of allosteric modulators. In each patch the open lifetime (D) was determined as $1 / \alpha$, the closed lifetime $(E)$ was determined as $1 / \delta$, while the flipped lifetime $(F)$ was $1 /(\beta+\gamma)$. Isoflurane increased the open lifetime compared with the taurine-alone condition. Data are shown as mean + S.E.M. of $5-8$ patches. $* P<0.05$. 
open dwell times, while both compounds decreased the likelihood that channels will adopt the longest-lived intracluster channel closed state. These effects seen with saturating concentrations of taurine are not seen when the modulators are coapplied with a saturating concentration of the more efficacious agonist glycine. As evidence accumulates for the physiologic role of the taurine-activated GlyR, it is important to note that the nature of allosteric modulation of these receptors can differ depending on the agonist activating the receptors.

\section{Authorship Contributions}

Participated in research design: Kirson, Todorovic, Mihic.

Conducted experiments: Kirson, Todorovic.

Performed data analysis: Kirson, Todorovic, Mihic.

Wrote or contributed to the writing of the manuscript: Kirson, Todorovic, Mihic.

\section{References}

Albrecht J and Schousboe A (2005) Taurine interaction with neurotransmitter receptors in the CNS: an update. Neurochem Res 30:1615-1621.

Badanich KA, Mulholland PJ, Beckley JT, Trantham-Davidson H, and Woodward JJ (2013) Ethanol reduces neuronal excitability of lateral orbitofrontal cortex neuron via a glycine receptor dependent mechanism. Neuropsychopharmacology 38 $1176-1188$

Baer K, Waldvogel HJ, Faull RL, and Rees MI (2009) Localization of glycine receptors in the human forebrain, brainstem, and cervical spinal cord: an immunohistochemical review. Front Mol Neurosci 2:25.

Choe KY, Olson JE, and Bourque CW (2012) Taurine release by astrocytes modulates osmosensitive glycine receptor tone and excitability in the adult supraoptic nucleus. J Neurosci 32:12518-12527.

Dahchour A, Quertemont E, and De Witte P (1996) Taurine increases in the nucleus accumbens microdialysate after acute ethanol administration to naive and chronically alcoholised rats. Brain Res 735:9-19.

Eggers ED and Berger AJ (2004) Mechanisms for the modulation of native glycine receptor channels by ethanol. J Neurophysiol 91:2685-2695.

Ericson M, Chau P, Clarke RB, Adermark L, and Söderpalm B (2011) Rising taurine and ethanol concentrations in nucleus accumbens interact to produce dopamine release after ethanol administration. Addict Biol 16:377-385.

Ericson M, Molander A, Stomberg R, and Söderpalm B (2006) Taurine elevates dopamine levels in the rat nucleus accumbens; antagonism by strychnine. Eur $J$ Neurosci 23:3225-3229.

Franks NP and Lieb WR (1994) Molecular and cellular mechanisms of general anaesthesia. Nature 367:607-614.

Hamill OP, Marty A, Neher E, Sakmann B, and Sigworth FJ (1981) Improved patchclamp techniques for high-resolution current recording from cells and cell-free membrane patches. Pflugers Arch 391:85-100.

Jonsson S, Adermark L, Ericson M, and Söderpalm B (2014) The involvement of accumbal glycine receptors in the dopamine-elevating effects of addictive drugs. Neuropharmacology 82:69-75.
Jonsson S, Kerekes N, Hyytiä P, Ericson M, and Söderpalm B (2009) Glycine receptor expression in the forebrain of male AA/ANA rats. Brain Res 1305 (Suppl):S27-S36.

Jonsson S, Morud J, Pickering C, Adermark L, Ericson M, and Söderpalm B (2012)

Changes in glycine receptor subunit expression in forebrain regions of the Wistar rat over development. Brain Res 1446:12-21.

Kirson D, Cornelison GL, Philpo AE, Todorovic J, and Mihic SJ (2013) Physiological concentrations of zinc reduce taurine-activated GlyR responses to drugs of abuse. Neuropharmacology 75:286-294.

Kirson D, Todorovic J, and Mihic SJ (2012) Positive allosteric modulators differentially affect full versus partial agonist activation of the glycine receptor $J$ Pharmacol Exp Ther 342:61-70.

Lape R, Colquhoun D, and Sivilotti LG (2008) On the nature of partial agonism in the nicotinic receptor superfamily. Nature 454:722-727.

$\mathrm{Lu} \mathrm{Y}$ and Ye JH (2011) Glycine-activated chloride currents of neurons freshly isolated from the prefrontal cortex of young rats. Brain Res 1393:17-22.

Lynch JW (2004) Molecular structure and function of the glycine receptor chloride channel. Physiol Rev 84:1051-1095.

Malosio ML, Marquèze-Pouey B, Kuhse J, and Betz H (1991) Widespread expression of glycine receptor subunit mRNAs in the adult and developing rat brain. EMBO J 10:2401-2409.

Mascia MP, Machu TK, and Harris RA (1996a) Enhancement of homomeric glycine receptor function by long-chain alcohols and anaesthetics. $\mathrm{Br} J$ Pharmacol 119: 1331-1336

Mascia MP, Mihic SJ, Valenzuela CF, Schofield PR, and Harris RA (1996b) A single amino acid determines differences in ethanol actions on strychnine-sensitive glycine receptors. Mol Pharmacol 50:402-406

Molander A, Lidö HH, Löf E, Ericson M, and Söderpalm B (2007) The glycine reuptake inhibitor Org 25935 decreases ethanol intake and preference in male Wistar rats. Alcohol Alcohol 42:11-18.

Molander A, Löf E, Stomberg R, Ericson M, and Söderpalm B (2005) Involvement of accumbal glycine receptors in the regulation of voluntary ethanol intake in the rat. Alcohol Clin Exp Res 29:38-45.

Mori M, Gähwiler BH, and Gerber U (2002) $\beta$-Alanine and taurine as endogenous agonists at glycine receptors in rat hippocampus in vitro. $J$ Physiol 539:191-200.

Qin F, Auerbach A, and Sachs F (2000a) A direct optimization approach to hidden Markov modeling for single channel kinetics. Biophys J 79:1915-1927.

Qin F, Auerbach A, and Sachs F (2000b) Hidden Markov modeling for single channel kinetics with filtering and correlated noise. Biophys J 79:1928-1944.

van den Pol AN and Gorcs T (1988) Glycine and glycine receptor immunoreactivity in brain and spinal cord. J Neurosci 8:472-492.

Waldvogel HJ, Baer K, Allen KL, Rees MI, and Faull RLM (2007) Glycine receptors in the striatum, globus pallidus, and substantia nigra of the human brain: an immunohistochemical study. J Comp Neurol 502:1012-1029.

Welsh BT, Goldstein BE, and Mihic SJ (2009) Single-channel analysis of ethanol enhancement of glycine receptor function. J Pharmacol Exp Ther 330:198-205.

Welsh BT, Kirson D, Allen HM, and Mihic SJ (2010) Ethanol enhances taurineactivated glycine receptor function. Alcohol Clin Exp Res 34:1634-1639.

Yamashita M, Ueno T, Akaike N, and Ikemoto Y (2001) Modulation of miniature inhibitory postsynaptic currents by isoflurane in rat dissociated neurons with glycinergic synaptic boutons. Eur J Pharmacol 431:269-276.

Yevenes GE and Zeilhofer HU (2011) Allosteric modulation of glycine receptors. $\mathrm{Br} J$ Pharmacol 164:224-236.

Address correspondence to: Dr. S. John Mihic, Department of Neuroscience, MC A4800, 2500 Speedway, University of Texas at Austin, Austin, TX 78712. E-mail: mihic@austin.utexas.edu 